\title{
HACIA UNA AUTÉNTICA INTEGRACIÓN CURRICULAR DE LAS TECNOLOGÍAS DE LA INFORMACIÓN Y COMUNICACIÓN
}

Francisco Javier Pariente Alonso

España

Cualquier plan de actuación educativa respecto a las Tecnologías de la Información y Comunicación ha de encuadrarse en un marco teórico que suponga la elección de una u otra forma de asumir la Tecnología Educativa.

El concepto de Tecnología Educativa ha experimentado en muy poco tiempo profundos cambios. Evolución cuyo origen hay que buscar tanto en el vertiginoso desarrollo tecnológico que caracteriza a la sociedad de nuestro tiempo, como en los cambios que se han producido en las ciencias que la fundamentan. En un periodo no excesivamente dilatado de tiempo podemos encontrar diversas y variadas concepciones que han originado perspectivas y prácticas docentes muy diferenciadas.

Se hace necesario, en definitiva, llegar a una aproximación conceptual de la Tecnología Educativa acorde en las finalidades educativas, y congruente con la sociedad actual, que sirva de base y justifique el desarrollo de cualquier plan de actuación relacionado con la incorporación e integración de las Tecnologías de la Información y Comunicación en Educación.

La Tecnología Educativa debe suponer una respuesta teórico-práctica que nos permita el diseño, análisis, selección, aplicación y evaluación coherentes de los recursos tecnológicos aplicados a los procesos de enseñanza-aprendizaje.

Es fundamental que los docentes dispongan de un conocimiento teórico justificado en la investigación-acción y la experiencia que les permita el desarrollo coherente de prácticas educativas adecuadas a los nuevos entornos, materiales y formatos. Estas prácticas habrán de suponer integrar el uso de las TIC con el fin de promover procesos de enseñanzaaprendizaje en y para la sociedad de la información.

Por tanto, la práctica educativa a la que nos referimos, implica necesariamente una integración curricular de las TIC, que ha de llevarse a cabo desde tres perspectivas distintas, una la consideración del conjunto de destrezas y competencias que suponen el uso de las TIC para profesores y alumnos. Otra como potente recurso en manos del profesor, medio eficaz y motivador de presentación y tratamiento de la información orientado a fomentar procesos de organización cel conocimiento del mundo que tiene el alumno. Y la tercera como agente de cambio, por el impacto que suponen respecto a los modos de acceder al conocimiento, al intercambio de información y a la metodología de los procesos de enseñanzaaprendizaje. Esta práctica comportará actuaciones docentes distintas pero no excluyentes, todo lo contrario, que abarcan desde la elaboración de materiales curriculares hasta su ensayo en la práctica, evaluación y posterior implementación. Es necesario que este proceso sea intencional y planificado; se trata de diseñar situaciones mediadas de aprendizaje concretas, en las que intervengan como elementos del proceso: el diseño, el análisis, la selección, aplicación y evaluación de los recursos tecnológicos centrados en el aprendizaje. 
Parece existir un consenso social generalizado en que en el mundo en que vivimos es tan necesario dominar las herramientas básicas de estas nuevas tecnologías, destrezas y competencias en el uso de las TIC, como saber leer, escribir y contar. Pero la realidad es que mientras nos planteamos en niveles teóricos si es necesario pasar del aula de informática a informatizar las aulas, las nuevas tecnologías no han conseguido "entrar" en el sistema educativo más allá de lo que significa disponer en los centros de un aula donde llevar a los alumnos a "jugar" una vez por semana, en el caso de le Educación Primaria o de haber creado un pequeño reino de taifas informatizado para los profesores de Tecnología en el caso de la Educación Secundaria.

Es necesario tomar conciencia de una vez por todas, y adoptar una serie de medidas que vayan más allá de la dotación de ordenadores para conseguir que la incorporación plena de las TIC a los centros educativos contribuya de manera decidida en la mejora del proceso de enseñanza y aprendizaje. Estas medidas implican políticas que han de contemplar de manera preferente y decidida:

- La formación del profesorado

- Orientaciones curriculares que supongan una verdadera integración de las TIC, que signifiquen la adecuación de las prácticas educativas a los nuevos entornos, medios y formatos. El colofón de estas orientaciones ha de materializarse en un auténtico diseño curricular de las TIC incardinado en el resto del currículum.

- La elaboración de materiales en distintos soportes para desarrollar al máximo sus potencialidades

- Mejora de la gestión integral de los centro

En mi opinión, la solución pasaría por conseguir que los centros llegaran a planes descentralizados y contextualizados que supusieran el desarrollo curricular de las TIC con carácter transversal en las distintas áreas instrumentales.

Esta propuesta se traduce en forma de distintas implicaciones para cada uno de los componentes que conforman el proceso educativo:

a) Respecto a los alumnos podemos señalar tres objetivos fundamentalmente:

1) El desarrollo de capacidades dirigidas a construir el conocimiento mediante entornos que faciliten:

- Un aprendizaje significativo

- El trabajo en equipo

- Situaciones comunicativas

- Creatividad y autonomía (capacidad para resolver problemas y actuar autónomamente.)

2) La adquisición de destrezas y habilidades relacionadas con la búsqueda, selección, análisis y organización de la información y el dominio funcional de las tecnologías de la información y la comunicación

3) El uso racional, critico y participativo de las TIC capacitando al alumno para:

- Acceder a la información 
- Tratarla y experimentar con ella

- Establecer relaciones y asociaciones

- Expresarse con ella

b) Respecto al profesorado cabría apuntar:

1) Se hace necesario poner a disposición del profesorado, espacios de reflexión sobre el uso de las TIC que le lleve a identificar con claridad los modelos pedagógicos y didácticos sobre los que quiere aplicar estas tecnologías

2) Es imprescindible mejorar la competencia del profesorado en la utilización de TICs para organizar, incorporar y desarrollar estrategias de enseñanza en el nuevo contexto de la sociedad de la información.

3) Es indispensable la utilización de las TIC como un medio eficaz y motivador de presentación y tratamiento de la información orientado a fomentar procesos de organización del conocimiento del mundo que tiene el alumno.

4) Una de las principales características del profesorado es su heterogeneidad, tanto en la competencia de las herramientas informáticas como en su experiencia desde el punto de vista educativo. Deben plantearse soluciones desde el ámbito de la Formación del profesorado ( inicial y permanente) que recojan el paso cronológico por un itinerario compuesto por cinco estadios:

- Alfabetización en el uso de las TIC. (Destrezas en la utilización de soft y hard)

- Capacitación didáctica.

- Adaptación de materiales.

- Producción de materiales.

- Evaluación

c) Respecto al currículo, se ha de conseguir

- Integrar las TIC en cada área instrumental de forma transversal.

- Confeccionar de un diseño curricular de las TIC que suponga el desarrollo del punto anterior

Como todo desarrollo curricular, el de las TIC, desde esta perspectiva transversal a las áreas instrumentales, debería tender a contemplar los siguientes aspectos:

- La explicitación consensuada del propio plan

- Una fundamentación teórica o marco teórico común

- Selección y secuenciación de contenidos y relación con los contenidos de las áreas instrumentales

- Criterios de tratamiento

- Temporalización 
- Supuestos metodológicos

- Criterios de organización espacio-temporal

- Establecer los materiales didácticos a utilizar

- Diseño de actividades

- Evaluación

d) Incluso podríamos señalar, respecto a los centros, dos finalidades:

- El fomento y desarrollo de la comunicación exterior, es decir con el entorno en general y con la Comunidad Educativa en particular; e interior, esto es, a través del acceso y gestión de datos, documentos, servicios y en general de la gestión administrativa global del centro.

- Facilitar una mejor organización y gestión de servicios y recursos

Otra medida de suma importancia, en consonancia con lo anteriormente expuesto, es contemplar la función específica del tecnólogo educativo, como profesional conocedor de los procesos comunicativos de enseñanza y aprendizaje, de los entornos educativos y de sus necesidades, capacitado para impulsar, como figura interna en lo centros, todo este proceso y elaborar propuestas abiertas y contextualizadas que consideren la experiencia de los profesores y apoyen sus actuaciones con los alumnos. En cualquier caso, hoy en día el conocimiento y uso de medios didácticos y recursos tecnológicos en general resulta imprescindible para todos los profesionales de la educación, con independencia del nivel educativo en el que desarrollen su actividad. Ahora bien, la figura del tecnólogo educativo, se hace imprescindible si pensamos que lo que se trata es de disponer en los centros de auténticos especialistas en diseñar, aplicar y evaluar estrategias de intervención educativa (planificación, organización, orientación...) que consideren la utilización de recursos tecnológicos y, a su vez, organizar y planificar los recursos tecnológicos de las instituciones educativas para su máximo aprovechamiento.

\section{REFERENCIAS BIBLIOGRÁFICAS}

AREA MOREIRA, M. La tecnología educativa y el desarrollo e innovación del curriculum en http://www.ull.es/publicaciones/tecinfedu/TE1.htm (Ponencia presentada en el XI CONGRESO NACIONAL DE PEDAGOGÍA. San Sebastián ) 1996

AREA MOREIRA, M. La educación para los medios de comunicación. Apuntes para una política de integración curricular. Revista de Medios y Educación, o 7, junio, 1998 (PixeBit) en www.sav.us.es/pixelbit/pixelbit.htm

BARTOlOMÉ PINA, A. Preparando para un nuevo modo de conocer. En http://www.ull.es/departamentos/didinv/tecnologiaeducativa/doc-bartolome.htm

CABERo Almenara, J. Nuevas tecnologías, comunicación y educación. En EDUTEC. REVISTA ELECTRÓNICA DE TECNOLOGíA EDUCATIVA, 1996, http://www.uib.es/depart/gte/revelec1.html

GALlEgo ARUFAT, Mํ. J. El ordenador, el curriculum y la evaluación de software educativo. Proyecto Sur ediciones Granada 1994 
MARQUeS GRAells, P. La tecnología educativa: Conceptualización, líneas de investigación en http://dewey.uab.es/pmarques/uabinvte.htm 1999 (última revisión: 30/08/04)

MEC. Una educación de calidad para todos y entre todos. Propuestas para el debate" En http://debateeducativo.mec.es. 2004

VV AA: "La Educación que queremos. Seminarios de primavera 2001 ¿Qué podemos esperar de las Tecnologías de la Información?" Ed. Fundación Santillana. Madrid 2001

ZABALZA, M.A. (1988). Diseño y desarrollo curricular.: Narcea. Madrid. 1987

ZUBERO BEASKOETXEA, I Participación y democracia ante las nuevas tecnologías. Retos políticos de la sociedad de la información.

En http://www.uned.es/ntedu/espanol/master/primero/modulos/tecnologia-y-sociedad/participacion-ydemocracia.htm 


\title{
Contactar
}

Revista lberoamericana de Educación

\author{
Principal OEI
}

